\title{
Sema4C induces the endothelial-mesenchymal transition of tumor-associated lymphatic endothelial cells in cervical cancer via the ERK pathway
}

\section{Jin Peng}

Shandong University Qilu Hospital

Xijiang Liu

Shandong Provincial Hospital

Chengcheng $\mathrm{Li}$

Shandong University Qilu Hospital

\section{Min Gao}

Shandong University Qilu Hospital

Hongyan Wang ( $\square$ hongyanwang@sdu.edu.cn )

Shandong University Cheeloo College of Medicine https://orcid.org/0000-0002-0923-0932

\section{Primary research}

Keywords: Sema4C, primary tumor associated lymphatic endothelial cells, endothelial-mesenchymal transition, ERK

Posted Date: August 21st, 2020

DOl: https://doi.org/10.21203/rs.3.rs-52892/v2

License: (c) (i) This work is licensed under a Creative Commons Attribution 4.0 International License. Read Full License 
1 Sema4C induces the endothelial-mesenchymal transition of tumor-associated

2 lymphatic endothelial cells in cervical cancer via the ERK pathway

3

4 Jin Peng ${ }^{1 \#}$ Xijiang Liu ${ }^{2 \#}$ Chengcheng Li $^{1}$ Min Gao $^{1}$ Hongyan Wang ${ }^{1 *}$

5

$6{ }^{1}$ Department of Obstetrics and Gynecology, Qilu Hospital, Cheeloo College of

7 Medicine, Shandong University, Jinan, 250001, China

$8 \quad{ }^{2}$ Department of Anesthesiology, Shandong Provincial Hospital affiliated to Shandong

9 First Medical University, Jinan 250021, China

$10 \quad$ \#These authors contribute equally to this work.

11

12

13

14

*Corresponding Author

Hongyan Wang

Department of Obstetrics and Gynecology,

Qilu Hospital, Cheeloo College of Medicine,

Shandong University

No.107 West Wenhua Road, Jinan, 250001, China

Tel:+86-0531-88361855

Fax:+86-0531-86927544

E-mail: hongyanwang@sdu.edu.cn 


\section{Abstract}

Background: The endothelial-mesenchymal transition (EndMT) is a process that increases the promigratory abilities of endothelial cells (ECs). Although lymphatic endothelial cells (LECs) plays a positive role in tumor lymphatic metastasis, the regulation of LECs undergoing EndMT remains poorly understood. Previous study indicated that Semaphorin 4C (Sema4C) could be a marker of LECs in cervical cancer. Here, we try to understand the mechanism that Sema4C could promote LECs to get mesenchymal characters and enhance lymphatic metastasis in cervical cancer.

Methods: The co-location of Sema4C and lymphatic marker LYVE1 was verified by confocal laser scanning. Primary tumor-associated LECs (TLECs) were distinguished from cervical cancer by flow cytometry from a mouse xenograft cervical tumor model. The promigratory ability was assessed using the Transwell test. Lentivirus infection was used to alter the expression of Sema4C in TLECs and the infection efficiency was tested by confocal laser scanning. The phospho-extracellular signal-regulated kinase (p-ERK) signaling pathway was measured using the ERK inhibitor (PD98059) ,as well as quantitative PCR and Western blot analysis.

Results: Sema4C and LYVE1 co-located on TLECs. Primary TLECs were successfully separated and cultured. Overexpressing Sema4C by lentivirus infection stimulated the invasive capacity of TLECs and downregulated E-cadherin, whereas knocking down Sema4C had the opposite effects. TLECs pro-migratory ability was found to depend on the extracellular signal-regulated kinase (ERK) signaling pathway, as the ERK inhibitor (PD98059) reversed Sema4C-induced E-cadherin expression and migration ability.

Conclusion: The results of this study suggest that the Sema4C-ERK-E-cadherin pathway appears to be critical for the EndMT of TLECs, which might promote lymph 
node metastasis. Thus, Sema4C could be a promising target for the treatment of cervical cancer with lymphatic metastasis.

Keywords: Sema4C, primary tumor associated lymphatic endothelial cells, endothelial-mesenchymal transition, ERK.

\section{Background}

Lymphatic metastasis is one of the crucial routes of metastasis in cervical cancer (1). Treatment failure in cervical cancer is often due to lymph node metastasis (2). Lymphatic vessels are usually viewed as playing a passive role in metastasis, serving merely as a channel for tissue-invading tumor cells (3). Recent studies have focused on the immune-regulatory function of lymphatic endothelial cells (LECs), which are active players in lymphatic metastasis $(3,4)$. However, it remains unclear whether and how LECs actively regulate lymphatic metastasis in cancer.

The endothelial - mesenchymal transition (EndMT) is a process by which endothelial cells (ECs) display considerable plasticity in the transition to mesenchymal cells; this was originally observed during heart development, whereas recent studies have suggested its role in cancer and fibrosis. During this process, ECs lose their endothelial markers such as E-cadherin and cluster of differentiation and acquire a migratory phenotype, along with mesenchymal markers such as vimentin and fibroblast special protein $(5,6)$. LECs also express E-cadherin and can be induced to enter the epithelial - mesenchymal transition (EMT) by WNT5B and Kaposi's sarcoma-associated herpesvirus $(7,8)$. E-cadherin is a major component of epithelial cell junctions, as well as a hallmark of the EndMT (6). However, our current understanding of the regulatory mechanism underlying E-cadherin expression in EndMT is still limited. In the EMT, which is analogous to EndMT, epidermal growth factor is suggested to induce the downregulation of E-cadherin through the extracellular signal-regulated kinase (ERK) pathway (9), which might activate a similar signaling pathway in the EndMT.

Semaphorins (Semas) are a large family of extracellular signaling proteins that regulate the motility of cells during the development of nevi (Sema3A, 3F, 6C, 6D), the neuroendocrine system (Sema7A and Sema4D), the immune system (Sema4D), reproductive systems (Sema3), and cancer progression (Sema4D, 3A, 6D) (10-12). 
Interestingly, Semas antagonize the effect of vascular endothelial growth factor (VEGF) (13). In addition, several recent studies have suggested that Sema3F may affect lymphangiogenesis $(14,15)$. In previous study, TLECs were isolated and normal LECs using in situ laser capture microdissection, and determined that synthetic membrane-bound Sema4C functions as an autocrine factor that promotes lymphangiogenesis (16). Nevertheless, the mechanism of Sema4C in regulating TLECs biological characteristics is largely unknown.

It is well known that tumors or metastasis must rely, at least in part, on the gene regulatory events in cells that constitute the primary tumor, as well as the tumor microenvironment (TME) (17). LECs, which are one of the most important components in the TME, also have special gene expression compared with non-tumor LECs such as VEGFR3, which is activated under pathological situations including cancer and inflammation (18). LECs in the market are separated from human lymph nodes (Catalog \#2500; ScienCell Research Laboratories, Carlsbad, CA, USA). The differential expression of genes between normal LECs and TLECs prompted us to assess the TLECs from tumors. Flow cytometry has been proven to be the best way for isolating cells, as well as analyzing the TME (19). For the first time, primary TLEs separated from mouse cervical tumor were used in this study for LEC biological characterization.

This study evaluated the role of Sema4C in the transdifferentiation of primary TLECs to determine mesenchymal characteristics. We reported that Sema4C promotes TLECs invasiveness and regulates the ERK-E-cadherin pathway. We propose that this pathway is involved in EndMT-like development. Our findings indicate that the control of the Sema4C-ERK-E-cadherin pathway may be a novel target for cancer therapy, which may potentially inhibit endothelial transdifferentiation to mesenchyme-like cells, thereby blocking the lymphatic metastasis of cancer. This preclinical study provides new insights into Sema4C in TLECs and confirms the active participation of TLECs in lymphatic metastasis.

\section{Materials and Methods}

\section{Antibodies and cell line}

All reagents in this study are of analytical grade and commercially available. The primary antibodies used in this study were as follows: Sema4C antibody (sc- 
136445,Santa Cruz Biotech, USA); LYVE1 antibody for flow cytometry (53-043382,ebioscience,USA, $5 \mathrm{ul} / 5-8 \times 10^{6} \quad$ cells $\left./ \mathrm{ml}\right)$; LYVE1 antibody for immunohistochemistry ( ab14917,abcam, USA). Vascular endothelial growth factor receptor 3(VEGFR3, ab51874), E-cadherin(ab181296), ERK1/2(ab17942), pERK1/2(ab214362) and b-actin(ab8227) antibodies were from abcam, USA. The alkaline phosphatase-conjugated anti-rabbit(A9919), anti-mouse(A4312) were purchased from Sigma, USA. ERK inhibitor, PD98059 (HY-12028), was purchased from MCE, USA. Mouse cervical cancer cell line U14 was purchased from Yubo company, Shanghai (YB-M060). The cells were cultured in RPMI 1640 medium (Gibco-BRL, US) with 10\% (v/v) fetal bovine serum (Gibco-BRL, US).

\section{Lentivirus for delivery of full-length Sema4C and siRNA against Sema4C}

Overexpression and silencing Sema4C lentivirus were purchased from Western Biomedical technology, China. A full-length mouse Sema4C open reading frame was obtained by PCR, using cDNA as templates, the primers sequences were : forward $\left(5^{\prime}-\right.$

\section{AGGGTTCCAAGCTTAAGCGGCCGCGCCACCATGGCCCCACACTGGGCTGT} CTGG-3')

reverse $\left(5^{\prime}-\right.$ GATCCATCCCTAGGTAGATGCATTCATACTGAAGACTCCTCTGGGTTG-3'). Lentivirus for delivery of full-length Sema4C was LV5. The final construction was termed as LV5-Sema4C. The control construction was LV5NC. Lentivirus for delivery of siRNA against Sema4C was LV3.The Sema4C siRNA was 5'CCTATGCCTTCCAGCCCAA-3', termed as LV3-Sema4C siRNA, and the sequence for control siRNA (5'-TTCTCCGAACGTGTCACGT-3') named as LV3NC. The Sema4C siRNA had been verified in previous article (9).

\section{Animals}

C57BL/6 female mice were purchased from Tengxin Biomedical technology, Chongqing, China. The mice were maintained in the accredited animal facility of Shandong University. The animal room had a controlled temperature $\left(23-25^{\circ} \mathrm{C}\right)$, photoperiod reversed 12/12 h light/dark cycle with lights on from 8:00 to 20:00; and relative humidity $(50-60 \%)$. The hygienic status was specific pathogen-free (SPF) 
according to the Association for Assessment and Accreditation of Laboratory Animal Care International (Laboratory Animal Facility of Tsinghua University) recommendations. They were used at age 4-6 wk, 18-22 gram weight. All of the experiments carried out in accordance with the International Council for Laboratory Animal Science (ICLAS) considering the animal rights. All of the experimental protocols were approved by Animal Care and Use Commit of Shandong University for animal ethics.

\section{Mouse xenograft tumor model}

Animals were anesthetized using $2 \%$ isoflurane $/ \mathrm{O}_{2}$ flow in an induction chamber for $10 \mathrm{~min}$. Use the forceps to touch the leg of the mouse, confirm that the mouse is fully unconscious. Mice received $100 \mathrm{ul}\left(\approx 1 \times 10^{7}\right)$ of U14 cells s.c. into the left shoulder. After the tumors had grown to a palpable size below the skin surface $\left(\approx 15 \mathrm{~mm}^{3}, 4\right.$ weeks after injection), keep mice in their home cage for euthanasia, and put their cage in a chamber with $\mathrm{CO}_{2}$. Turn on the compressed $\mathrm{CO}_{2}$ for at least $20 \mathrm{~min}$ and $2 \mathrm{~L} / \mathrm{min}$. The percentage of the chamber volume displaced by minute by flow rate of $\mathrm{CO} 2$ was 20\%. Let the $\mathrm{CO}_{2}$ on until at least 1 min after mice stop breathing. Place the sacrificed-mouse on a polystyrene board, cut the tumor and measure it.

\section{Separating TLECs by flow cytometry}

Tumor tissues connective was break down by $1 \%$ collagenase IV for $30 \mathrm{~min}$, and $0.25 \%$ pancreatic enzymes for $5-8 \mathrm{~min}$. Cell suspensions was filtration through cell sieves, 200-m mesh. After incubation for $48 \mathrm{~h}$ at $37^{\circ} \mathrm{C}$ in $5 \% \mathrm{CO}_{2}$ for $48 \mathrm{~h}$, the suspend cells were removed. Cells were then incubated with the monoclonal antibody against mouse LYVE1-PE, after adjusting cell density as $5-8 \times 10^{6} / \mathrm{ml}$. The samples were analyzed on a FACSC alibur apparatus (BD Biosciences) and cells that expressed LYVE1 were separated. LECs were cultured in EBM-2 medium (LONZA, Switzerland) at $37^{\circ} \mathrm{C}$ in $5 \% \mathrm{CO}_{2}$ with $2 \%(\mathrm{v} / \mathrm{v})$ fetal bovine serum (Gibco-BRL, US).

\section{Immunohistochemistry for quantification of lymphatic microvessel density (LMVD)}

An immunohistochemical analysis of LYVE1 was performed using the avidin-biotinperoxidase complex method. Dewaxed and rehydrated mouse tumor tissue sections 
were incubated overnight at $4^{\circ} \mathrm{C}$ with a rabbit monoclonal anti-mouse LYVE1 antibody at a 1:100 dilution or with PBS for negative control, then washed with PBS. Biotinylated anti-rabbit immunoglobulin (DAKO, Kyoto, Japan) was then added to the sections for 30 minutes at room temperature. Peroxidase-conjugated avidin (DAKO, Kyoto, Japan) was then applied after the sections were washed with PBS. The peroxidase activity was detected by exposing the sections to a solution of $0.05 \%$ 3, 3-diaminobenzidine and $0.01 \% \mathrm{H}_{2} \mathrm{O}_{2}$ in Tris- $\mathrm{HCl}$ buffer (3, 3-diaminobenzidine solution) for 10 minutes at room temperature. The sections were counterstained with hematoxylin.

The stained sections were then analyzed using standard light microscopy (Nikon, Eclipse 200). Under low magnification, the most vascularized intratumoral areas were chosen (hot spots). The number of immunostained lymphatic vessels found in 3 hot spot areas at $400 \times$ magnification were counted. The LMVD for each case was expressed by the mean value (total number of vessels in 3 hot spot microscopic fields/3).

\section{Confocal microscopy imaging}

For immunofluorescence staining, frozen tissue sections were used. The first antibody was applied to the slides at $4^{\circ} \mathrm{C}$ overnight (LYVE1: 1:50, Sema4C: 1:20). After the incubation with the first antibody, the cells were washed three times with cold PBS and incubated with FITC- or Cy3-conjugated IgG at a 1:50 dilution in PBS for $30 \mathrm{~min}$ at room temperature. The nuclei were stained for $5 \mathrm{~min}$ at room temperature with DAPI. The cells were rinsed with PBS and observed using a confocal microscope (Olympus, Japan).

\section{Immunohistochemistry for identification of TLECs}

An immunohistochemical identification analysis of LECs was performed using the avidin-biotin-peroxidase complex method. LECs were incubated on sheet glasses in 12 -well plate and fixed by $4 \%$ paraformaldehyde for $20 \mathrm{~min}$ at room temperature. The cells were then incubated overnight at $4^{\circ} \mathrm{C}$ with polyclonal anti-mouse VEGFR3 antibody at a 1:30 dilution and then washed with PBS. HRP-immunoglobulin (abcam, USA) was then added to the sections for 30 minutes at $37^{\circ} \mathrm{C}$. After the sections were washed with PBS, peroxidase-conjugated avidin (DAKO) was then applied. The 
peroxidase activity was detected by exposing the sections to a solution of $0.05 \% 3,3$ diaminobenzidine and $0.01 \% \mathrm{H}_{2} \mathrm{O}_{2}$ in Tris- $\mathrm{HCl}$ buffer (3, 3-diaminobenzidine solution) for 10 minutes at room temperature. The sections were counterstained with hematoxylin.

\section{Cell transfection}

The primary TLECs were cultured in 24-well tissue culture plates or flasks at $37^{\circ} \mathrm{C}$ with $5 \% \mathrm{CO}_{2}$ in a humidified incubator (Heraeus, Germany). For the lentivirus infection, the cells $\left(10^{5} / \mathrm{ml} / 200 \mathrm{ul}\right)$ were added with lentivirus at $\mathrm{MOI}=40$, when the cells were $70 \%$ confluent according to the preliminary experiment. The cells were infected for $72 \mathrm{hrs}$ for the next experiments. The control of lentivirus for delivery fulllength LV5-Sema4C is LV5control (LV5NC), while the control of LV3-siRNA Sema4C is LV3control (LV3NC). The package system is four plasmid system, LV5GFP/LV3-GFP, PG-p1-VSVG, PG-REV, PG-P3-RRE. The results of transfection were detected using a confocal microscope (Olympus, Japan).

\section{Quantitative Real-time PCR}

For quantitative real-time PCR, total RNA was isolated from cell lines in Trizol reagent (Invitrogen, CergyPontoise, France). First-strand cDNA synthesis was performed using a cDNA synthesis kit (Amersham Bioscience, NJ). Quantitative realtime PCR was performed on an ABI PRISM 7700 Sequence Detection System (Applied Biosystems, CA) by using a QuantiTect SYBR Green kit (Qiagen, CA). The primers were designed using Primer Express 3.0 software, identified by Basic Local Alignment Search Tool (BLAST) (http://www.ncbi.nlm.nih.gov/BLAST) and purchased from Invitrogen. Each sample was run in triplicate. Conditions for quantitative PCR reaction were as follows, one cycle of $50^{\circ} \mathrm{C}$ for $15 \mathrm{~min}$, one cycle of $94^{\circ} \mathrm{C}$ for 4 min, 35 cycles of $94^{\circ} \mathrm{C}$ for $20 \mathrm{~s}, 60^{\circ} \mathrm{C}$ for $30 \mathrm{~s}$, and $70^{\circ} \mathrm{C}$ for $35 \mathrm{~s}$. At the end of the PCR reaction, samples were subjected to a melting analysis to confirm specificity of the amplification. The Sema4C primer sequences were: Forward: CCTCCCATCTGTATGTCTGCG ; Reverse: GCTGGGTCATATGGGCATTTAC . For internal standard, the actin primer sequences were: Forward: GAGACCTTCAACACCCCAGC; Reverse: ATGTCACGCACGATTTCCC.

\section{Immunoblotting}


For the Western blot analyses, the cells were lysed in RIPA buffer (50 mM Tris/ $\mathrm{HCl}$, $\mathrm{pH} 7.2,150 \mathrm{mM} \mathrm{NaCl}, 1 \% \mathrm{NP}-40,0.1 \% \mathrm{SDS}$, and $0.5 \%$ (w/v) sodium deoxycholate). Equivalent amounts of the cell extracts $(150 \mathrm{mg})$ were separated on a $10 \%$ sodium dodecyl sulfate polyacrylamide gel (SDS-PAGE) and transferred onto a polyvinylidenedifluoride membrane (PVDF). The membranes were blocked in $25 \mathrm{mM}$ Tris ( $\mathrm{pH} 8.0$ ) containing $125 \mathrm{mMNaCl}, 0.1 \%$ Tween 20, and 5\% skim milk for 1 hour and then incubated with the diluted primary antibodies (Sema4C: 1:500, E-cadherin, ERK1/2 and p-ERK1/2: $1: 2000$ and $\beta$-actin: 1:1000) at $4^{\circ} \mathrm{C}$ overnight. After incubation with the primary antibodies, the secondary antibodies were added at a 1:1000 dilution. The immune reactive bands were visualized with Enhanced chemiluminescence (ECL) western-blot technique.

\section{The migration assay}

For the migration assay, $5 \times 10^{3}$ cells were seeded in $100 \mathrm{ml}$ EBM- 2 media on the top of polyethylene terephthalate (PET) membranes within transwell cell culture inserts (24-well inserts, $8 \mathrm{~mm}$ pore size; Corning Life Sciences, Corning, NY). The bottom chamber was filled with $600 \mathrm{ml}$ EBM-2 media containing 2\% FBS and the supernatant from NIH3T3 cells (mouse embryonic fibroblast cell line) to act as a chemotactic factor (CF). The cells were incubated for $48 \mathrm{hrs}$ at $37^{\circ} \mathrm{C}$ with $5 \% \mathrm{CO}_{2}$. Subsequently, the cells were fixed in $2.5 \%(\mathrm{v} / \mathrm{v})$ glutaraldehyde and stained with crystal violet. Cells on the gel bottom were visualized under a microscope (Leica, Germany) and quantified by counting the number of cells in three randomly chosen fields at a 100-fold magnification.

\section{Statistical analysis}

Data were expressed as mean \pm SEM and statistical analysis was performed using SPSS version 13.0 software. Statistical comparisons include one-way ANOVA followed by post hoc comparison (Dunnett test and LSD-test).

\section{Results}

\section{Separation of TLECs from a mouse xenograft cervical tumor model}

Firstly, we determined the expression of Sema4C on TLECs in this mouse cervical tumor tissue. Using a confocal microscope, LYVE1 was used to detected lymphatic vessels, and co-location (merge) was found between Sema4C (green) and LYVE1 
(red) (Fig. 1A). We employed primary TLECs to maintain the entire TME of cervical tumors, including the immune and growth conditions, as well as interactions among cells using BALB/C mice with U14 tumor allografts. The U14 cells were injected subcutaneously (s.c.) into the left shoulder and lymphatic microvessel density was tested by LYVE1 immunohistochemistry for the most appropriate time point to separate TLECs (Fig. 1B ). By LMVD analysis (Fig.1C), after tumor growing to the $7^{\text {th }}$ day, the lymphatic vessels became intensively. After 14 days, massive necrosis happened and the density of lymphatic vessels was decreased significantly. Then the appropriate time for separation of TLECs was the $7^{\text {th }}$ day after tumor cells injection. LYVE1-positive cells were separated by flow cytometry (Fig. 1D). The cells were cultured successfully, appeared as a typical monolayer (Fig.1E ). VEGFR3 was further used to identify the separated cells (Fig. 1F), and most of the cells were positive for VEGFR3. Thus, the separated cells were both LYVE1 and VEGFR3 positive cells.

\section{Sema4C regulates the migration ability of TLECs}

We regulated the expression of the target gene Sema4C via lentivirus infection,noninfected cells was used as blank controls (Fig. 2A). Small interfering RNA (siRNA) Sema4C lentivirus (LV3-siRNA) and full-length Sema4C lentivirus (LV5-Sema4C), as well as control lentivirus (LV3NCand LV5NC), all expressed green fluorescent protein (GFP) at a multiplicity of infection of 40MOI for $72 \mathrm{~h}$. The efficiency of the silencing lentivirus and the overexpression lentivirus infection was $90 \%$ according to the fluorescence test. Quantitative PCR (qPCR) showed almost 0.3- and 3.5-fold higher RNA levels in LV3-siRNA and LV5-Sema4C relative to the control, respectively (Fig. 2B). We then studied the effects of Sema4C on the invasiveness of the TLECs using a classic Transwell model. The quantity of cells at the bottom of the membrane, which reflects the migration of cells, was $56 \pm 8.61,52 \pm 7.38,51.2 \pm 5.89$, $40 \pm 8.16$, and $73 \pm 12.78$ for con, LV3NC-siRNA-, LV5NC-siRNA-, LV3-siRNA-, and LV5-Sema4C-treated cells, respectively (Fig. 2C). Silencing Sema4C inhibited the migratory ability of TLECs and induced the overexpression Sema4C.

\section{Sema4C regulates EndMT of TLECs}

The migratory ability of ECs facilitates cellular movement similar to mesenchymal cells (i.e., EndMT). Based on the previously mentioned literature, a possible 
signalling pathway in the EndMT was investigated. Sema4C inhibited the expression of E-cadherin, whereas silencing of Sema4C promoted expression. Moreover, the level of phosphorylated ERK (p-ERK1/2), the active form of ERK1/2, was altered with lentivirus infection of Sema4C full-length gene or Sema4C siRNA. Sema4C stimulated the phosphorylation of ERK. A similar pattern was observed for mRNA and protein levels using qPCR (Fig. 3A) and western blotting (Fig. 3B and 3C), respectively.

\section{The ERK inhibitor reverses the Sema4C-induced EndMT}

We hypothesized that Sema4C regulates the EndMT partially through the regulation of E-cadherin, which is one of the most important characteristics of EndMT. The ERK inhibitor, PD98059 (30 mM), was employed to determine the alterations in migration ability after Sema4C silencing or overexpression by lentivirus infection. The results showed that PD98059 effectively enhanced the inhibition of migration ability of Sema4C siRNA infection comparing to control group (Fig.4A). Furthermore, the addition of PD98059 partially relieved the induced migration ability of TLECs after overexpression of Sema4C (Fig. 4A). The quantities of cells at the bottom of the membrane, as shown in the bar on the right side, reflected the migration of the cells, which were $56 \pm 8.61,40 \pm 8.16,73 \pm 12.78,18 \pm 4.56$, and $58 \pm 8.87$, for con, LV3siRNA-, LV5-Sema4C-, LV3-siRNA+PD98059-, and LV5-Sema4C+PD98059treated cells, respectively (Fig. 4B).

\section{The ERK inhibitor stimulates E-cadherin expression by blocking Sema4C}

The ERK inhibitor was used to determine whether Sema4C-mediated regulation of Ecadherin depends on the phosphorylation level of ERK1/2. While silencing Sema4C inhibited the phosphorylation level of ERK, the introduction of PD98059 further enhanced the upregulation of E-cadherin expression by Sema4C siRNA infection. However, inhibition of phosphorylation of ERK1/2 partially neutralized the downregulation of E-cadherin that was regulated by Sema4C. The RNA levels of Sema4C and E-cadherin were in accordance with the protein levels (Fig. 5A, 5B, 5E, and $5 \mathrm{~F}$ ). Because the phosphorylation of ERK1/2 is a special characteristic of ERK1/2 at the protein level, we also tested the RNA levels of ERK1/2 (Fig. 5C). Recovery of p-ERK1/2 expression in LV5-Sema4C plus PD98059 was observed compared to LV5-Sema4C alone, indicating that PD98059 can effectively block Sema4C (Fig. 5D 
and 5G). ERK1/2 RNA expression has increased after Sema4C overexpression, but ERK1/2 protein level did not change significantly (Fig. 5H and 5I). However, it is interesting that protein level of phosphorylation ERK1/2 show the same tendency as ERK1/2 RNA level. Thus, phosphorylation level of ERK1/2 is more important in Sema4C signaling pathway, rather than ERK1/2.

\section{Discussion}

Tumor-associated lymphangiogenesis is a key modulator of tumor metastasis, although the underlying mechanism remains unclear. It was recently shown that the lymphatic system actively participates in tumor metastasis(20), and that LECs play important roles in inducing immune tolerance(21). The ability of LECs to promote immunosuppression may induce tumor cell metastasis (22). A previous study using in situ laser capture microdissection showed that the gene expression profile of human tumor LECs differed from that of normal LECs, which was associated with increased tube formation ability, and found that Sema4C was differentially expressed (19). Here, we have focused on LECs undergoing EndMT to attain invasion ability, which was, at least in part, due to the higher expression of Sema4C in tumor LECs.

EndMT was initially discovered as an essential step in heart development. Further studies have demonstrated that EndMT occurs in cancer and tissue fibrosis (23), while the regulation of this process requires further studies. Furthermore, as one of the most important components of endothelial cells, whether and how LECs participate in the EndMT during cancer development remains unclear. The biological characteristics of LECs markedly change in tumors in vivo (17). Thus, in this study, we separated TLECs from mouse cervical tumor tissues by flow cytometry, instead of an LEC cell line from ScienCell, which was separated from normal lymphatic nodes. LYVE1 was used as the marker for LEC separation (19) and VEGFR3 as an identification marker (24).

It has been reported that some molecules show differences in molecular mechanism between their membrane and soluble forms. For example, full-length Sema3C is a tumor angiogenesis inhibitor, whereas cleaved Sema3C is a tumor progression promoter (25). The previous study showed that there were biological properties between the soluble and membrane forms of Sema4C (sSema4C and mSema4C). sSema4C promotes lymphagiogenesis, whereas mSema4C also directs cell-cell contacts, thereby providing the possibility of a new mechanism of mSema4C 
working on LECs (15). Thus, we used lentiviral transfection for consistent overexpression or silencing of mSema4C in TLECs.

In the previous study, Ras homolog family member A (RhoA) was critical to Sema4C-mediated signaling. One of distinct cell migration models, named amoeboidtype migration, is characterized by a spherical or elongated cell shape and is strongly dependent on Rho kinase activity (26). Thus, we presume that LECs migrate and undergo EndMT in the amoeboid-type, which could be promoted by Sema4C, as shown by the Transwell test. A spherical cell shape with a small number of short protrusions was found after passing through the polyethylene terephthalate membranes. Mechanistically, we found that the promotion of the migration ability of Sema4C-overexpressing LECs is in part attributable to ERK activation-induced repression of E-cadherin expression.

E-cadherin is a cell adhesive molecule that plays a key role in cellular adhesion and migration, which acts as one of the most important symbols of EndMT and can also be regulated by RhoA(27). A recent study showed a novel role for E-cadherin in regulating LEC progeny in newly synthesized lymphatic vessels $(25,28)$. In particular, forced disruption of E-cadherin-mediated intercellular adhesion opened the intercellular junctions in LEC monolayers as determined by another study using Transwell assays (29). Here, we showed that repression of E-cadherin is a necessary molecular event for the promotion of migration ability in Sema4C-overexpressing LECs. This finding is concordant with another study, which demonstrated that the overexpression of Sema4C suppresses E-cadherin, induces vimentin, and promotes fibronectin secretion in human kidney cells (30). Although our results indicate that Ecadherin is an important molecular target of Sema4C EndMT function, the downstream signaling pathway was impacted by the loss of E-cadherin expression, as well as promoted migrated ability, although the overexpression of Sema4C has yet to be determined. The expression of E-cadherin is an ERK-dependent mechanism. Hence, it will be interesting to determine whether the regulation of Sema4C-induced E-cadherin expression and migration ability in LECs are activated by ERK. The application of an ERK inhibitor has proven that Sema4C regulates E-cadherin, but this process is dependent on ERK activation.

\section{Conclusions}


In summary, we confirm that Sema4C expressed on TLECs by co-location with

411 LVYE1, and provide evidence that overexpression of Sema $4 \mathrm{C}$ is an important 412 molecular mechanism that contributes to the EndMT of TLECs by suppressing E413 cadherin expression. Mechanistically, the process involves the activation of the ERK 414 pathway, which functions upstream of E-cadherin and downstream of Sema4C. 415 Studies on TLECs are limited, and thus, it will be important to determine whether 416 enhanced ERK activation during the upregulation of Sema4C as observed in TLECs 417 can induce tumor lymphatic metastasis, which in turn, can be targeted using 418 pharmacological approaches.

\section{List of abbreviations}

421 Sema4C: Semaphorin 4C

EndMT: endothelial-mesenchymal transition

LECs: lymphatic endothelial cells

ECs: endothelial cells

TLECs: tumor-associated Lymphatic endothelial cells

ERK: extracellular signal-regulated kinase

EMT: epithelial-mesenchymal transition

Semas: semaphorins

VEGFR3: vascular endothelial growth factor receptor 3

VEGF: vascular endothelial growth factor

TME: tumor microenvironment

SPF: specific pathogen-free

LMVD: lymphatic microvessel density

SDS-PAGE: sodium dodecyl sulfate polyacrylamide gel

PVDF: polyvinylidenedifluoride membrane 
ECL: Enhanced chemiluminescence

437 PET: polyethylene terephthalate

438 CF: chemotactic factor

qPCR: Quantitative PCR

440

p-ERK1/2: phosphorylated ERK

RhoA: Ras homolog family member A

siRNA: small interfering RNA

GFP: green fluorescent protein

\section{Declarations}

446

\section{Ethics approval and consent to participate}

All of the experimental carried out in accordance with the International Council for

Laboratory Animal Science (ICLAS) considering the animal rights. All of the experimental protocols were approved by Animal Care and Use Committe of Shandong University for animal ethics.

\section{Consent for publication}

453 Not applicable

\section{Availability of data and materials}

456

The datasets used and/or analysed during the current study are available.

\section{Competing inter ests}

459 The authors declare that they have no competing interests

460

461 Funding

462 This work was supported by grants from the National Natural Science Foundation of 463 China (Nos. 81502238 and 81602286), and the Department of Medical and Health 464 Science Technology of Shandong province (No. 2016w0345), and from the 
465 Department of Science Technology of Jinan city (No. 201705051) and from the China

466 Postdoctoral Science Foundation (No. 2019T120594).

467

468 Authors' contributions

469 HW designed the experiments and wrote the paper. JP and XL carried out the 470 molecular experiments and analyzed the data and conceived the experiments; CL built 471 the mouse model,performed the histological examination of tumor, and separated the 472 cells; MG provided soft ware analysis. All authors read and approved the final 473 manuscript.

474

475 Acknowledgements

476 We would like show sincere appreciation to Qingliang Wang for the statistic 477 assistance on this study.

478

479 Authors' information

480 Jin Peng and Xijiang Liu contributed equally to this work. 
482 1. Frumovitz M. Sentinel Lymph Node Biopsy for Cervical Cancer Patients 483 What's It Gonna Take? Gynecol Oncol. 2017 Jan;144(1):3-4.

2. $\mathrm{Li} \mathrm{H}, \mathrm{Wu} \mathrm{X}$, Cheng $\mathrm{X}$. Advances in diagnosis and treatment of metastatic cervical cancer. J Gynecol Oncol. 2016 Jul;27(4):e43.

486 3. Hoshino A, Lyden D. Metastasis: Lymphatic detours for cancer. Nature. 2017 Jun 28;546(7660):609-10.

488 4. Lucas ED, Tamburini BAJ. Lymph Node Lymphatic Endothelial Cell 489 Expansion and Contraction and the Programming of the Immune Response. Front $490 \quad$ Immunol. 2019;10:36.

491 5. Saito A. EMT and EndMT: regulated in similar ways? J Biochem. 2013 492 Jun;153(6):493-5.

493 6. Kourtidis A, Lu R, Pence LJ, Anastasiadis PZ. A central role for cadherin 494 signaling in cancer. Exp Cell Res. 2017 Sep 1;358(1):78-85.

495 7. Wang SH, Chang JS, Hsiao JR, Yen YC, Jiang SS, Liu SH, et al. Tumour cell496 derived WNT5B modulates in vitro lymphangiogenesis via induction of partial 497 endothelial-mesenchymal transition of lymphatic endothelial cells. Oncogene. 2017 498 Mar;36(11):1503-15.

499 8. Lee HR, Li F, Choi UY, Yu HR, Aldrovandi GM, Feng P, et al. Deregulation 500 of HDAC5 by Viral Interferon Regulatory Factor 3 Plays an Essential Role in 501 Kaposi's Sarcoma-Associated Herpesvirus-Induced Lymphangiogenesis. mBio. 2018 502 Jan 16;9(1).

503 9. Wu H, Wang X, Liu S, Wu Y, Zhao T, Chen X, et al. Sema4C participates in 504 myogenic differentiation in vivo and in vitro through the p38 MAPK pathway. Eur J 505 Cell Biol. 2007 Jun;86(6):331-44.

506 10. Elder AM, Tamburini BAJ, Crump LS, Black SA, Wessells VM, Schedin PJ, 507 et al. Semaphorin 7A Promotes Macrophage-Mediated Lymphatic Remodeling during 508 Postpartum Mammary Gland Involution and in Breast Cancer. Cancer Res. 2018 Nov 509 15;78(22):6473-85.

510 11. Acker DWM, Wong I, Kang M, Paradis S. Semaphorin 4D promotes 511 inhibitory synapse formation and suppresses seizures in vivo. Epilepsia. 2018 512 Jun;59(6):1257-68. 
12. Ferreira GD, Capp E, Jauckus J, Strowitzki T, Germeyer A. Expression of semaphorin class 3 is higher in the proliferative phase on the human endometrium. Arch Gynecol Obstet. 2018 May;297(5):1175-9.

13. Sakurai A, Doci CL, Gutkind JS. Semaphorin signaling in angiogenesis, lymphangiogenesis and cancer. Cell Res. 2012 Jan;22(1):23-32.

14. Nasarre P, Gemmill RM, Drabkin HA. The emerging role of class-3 semaphorins and their neuropilin receptors in oncology. Onco Targets Ther. 2014;7:1663-87.

15. Doci CL, Mikelis CM, Lionakis MS, Molinolo AA, Gutkind JS. Genetic Identification of SEMA3F as an Antilymphangiogenic Metastasis Suppressor Gene in Head and Neck Squamous Carcinoma. Cancer Res. 2015 Jul 15;75(14):2937-48.

16. Wei JC, Yang J, Liu D, Wu MF, Qiao L, Wang JN, et al. Tumor-associated Lymphatic Endothelial Cells Promote Lymphatic Metastasis By Highly Expressing and Secreting SEMA4C. Clin Cancer Res. 2017 Jan 1;23(1):214-24.

17. Albini A, Mirisola V, Pfeffer U. Metastasis signatures: genes regulating tumor-microenvironment interactions predict metastatic behavior. Cancer Metastasis Rev. 2008 Mar;27(1):75-83.

18. Olmeda D, Cerezo-Wallis D, Riveiro-Falkenbach E, Pennacchi PC, ContrerasAlcalde M, Ibarz N, et al. Whole-body imaging of lymphovascular niches identifies pre-metastatic roles of midkine. Nature. 2017 Jun 28;546(7660):676-80.

19. Young YK, Bolt AM, Ahn R, Mann KK. Analyzing the Tumor Microenvironment by Flow Cytometry. Methods Mol Biol. 2016;1458:95-110.

20. Lukacs-Kornek V. The Role of Lymphatic Endothelial Cells in Liver Injury and Tumor Development. Front Immunol. 2016;7:548.

21. Humbert M, Hugues S, Dubrot J. Shaping of Peripheral T Cell Responses by Lymphatic Endothelial Cells. Front Immunol. 2016;7:684.

22. Ji RC. Lymph Nodes and Cancer Metastasis: New Perspectives on the Role of Intranodal Lymphatic Sinuses. Int J Mol Sci. 2016 Dec 28;18(1).

23. Cho JG, Lee A, Chang W, Lee MS, Kim J. Endothelial to Mesenchymal Transition Represents a Key Link in the Interaction between Inflammation and Endothelial Dysfunction. Front Immunol. 2018;9:294.

24. Harris AR, Perez MJ, Munson JM. Docetaxel facilitates lymphatic-tumor crosstalk to promote lymphangiogenesis and cancer progression. BMC Cancer. 2018 Jul 6;18(1):718. 
25. Mumblat Y, Kessler O, Ilan N, Neufeld G. Full-Length Semaphorin-3C Is an

548 Inhibitor of Tumor Lymphangiogenesis and Metastasis. Cancer Res. 2015 Jun $549 \quad 1 ; 75(11): 2177-86$.

550 26. Lawson CD, Ridley AJ. Rho GTPase signaling complexes in cell migration 551 and invasion. J Cell Biol. 2018 Feb 5;217(2):447-57.

552 27. Lee G, Kim HJ, Kim HM. RhoA-JNK Regulates the E-Cadherin Junctions of 553 Human Gingival Epithelial Cells. J Dent Res. 2016 Mar;95(3):284-91.

554 28. Connor AL, Kelley PM, Tempero RM. Lymphatic endothelial lineage 555 assemblage during corneal lymphangiogenesis. Lab Invest. 2016 Mar;96(3):270-82.

556 29. Hou WH, Liu IH, Tsai CC, Johnson FE, Huang SS, Huang JS. CRSBP557 1/LYVE-1 ligands disrupt lymphatic intercellular adhesion by inducing tyrosine 558 phosphorylation and internalization of VE-cadherin. J Cell Sci. 2011 Apr 15;124(Pt 559 8):1231-44.

560 30. Zhou QD, Ning Y, Zeng R, Chen L, Kou P, Xu CO, et al. Erbin interacts with 561 Sema4C and inhibits Sema4C-induced epithelial-mesenchymal transition in HK2 cells. 562 J Huazhong Univ Sci Technolog Med Sci. 2013 Oct;33(5):672-9. 


\section{Figure Legends}

Fig.1 Tumor-associated lymphatic endothelial cells (TLECs)were obtained from a mouse xenograft tumor. (A) The location of Sema4C was detected in mouse tumor tissue. In lymphatic vessels, Sema4C(green) have co-location with LYVE1(Red), one of the most notable marker for lymphatic endothelial cells. Nuclei were shown by DAPI staining (blue). (B)The most suitable time (the $7^{\text {th }}$ day after U14 cells injection) for separation of TLECs was determined by LYVE1 immunohistochemical analysis. (C) Quantification of the numbers of lymphatic vessels (LMVD) in $5^{\text {th }}, 7^{\text {th }}, 14^{\text {th }}$ days were measured. (D)Tumor tissues were dissociated into a cell suspension and then stained for LYVE1-PE. LYVE1-positive cells were separated by flow cytometry. (E) TLECs were cultured in EBM-2 medium for 5 days and 10 days. (F) VEGFR3positive TLECs were identified by immunohistochemistry.

Fig. 2 Role of Sema4C expression on migration ability of TLECs. (A) TLECs treated with lentivirus medium only (con), lentivirus vector control for Sema4C siRNA (LV3NC), lentivirus vector control for full-length Sema4C (LV5NC), Sema4C siRNA (LV3-siRNA), and full-length Sema4C (LV5-Sema4C). (B) TLECs with LV3siRNA or LV5-Sema4C were generated and confirmed by Sema4C mRNA expression as measured by real-time PCR. (C) The migration ability of cells (con, LV3NC,LV5NC,LV3-siRNA, and LV5-Sema4C) were assessed using a Transwell assay. (D) Quantification of the number of cells at the bottom of the Transwell chamber.

Fig. 3 Role of Sema4C expression on EndMT of TLECs. (A) Assessment of mRNA expression levels of Sema4C, E-cadherin, and ERK1/2 using quantitative PCR. (B) Protein expression levels of Sema4C, E-cadherin, p-ERK1/2, and total ERK (ERK1/2) using western blot analysis. (C) Quantification of protein expression levels of Sema4C, E-cadherin, p-ERK1/2, and total ERK (ERK1/2) using western blot. (D) Intensity for p-ERK1/2 was normalized to total ERK1/2 and represent as fold change over control group.

Fig. 4 The ERK inhibitor can reverse migration ability of TLECs induced by Sema4C. (A) The migration ability in TLECs was assessed using only the media of lentivirus (con), Sema4C siRNA (LV3-siRNA), full-length Sema4C (LV5-Sema4C), LV3-siRNA cultured with PD98059, and LV5-Sema4C cultured with PD98059. (B) Quantification of the number of cells from the bottom of the Transwell inserts was measured. 
599 Fig. 5 Sema4C stimulates EndMT of TLECs via the ERK pathway. (A-C) The 600 RNA expression levels of Sema4C, E-cadherin, and total ERK (ERK1/2) in TLECs 601 treated with only media of lentivirus (con), Sema4C siRNA (LV3-siRNA), full-length 602 Sema4C (LV5-Sema4C), LV3-siRNA cultured with PD98059, and LV5-Sema4C 603 cultured with PD98059. (D) The protein expression levels of Sema4C, E-cadherin, p604 ERK1/2, and total ERK (ERK1/2) in TLECs treated with only media of lentivirus 605 (con), Sema4C siRNA (LV3-siRNA), full-length Sema4C (LV5-Sema4C), LV3606 siRNA cultured with PD98059, and LV5-Sema4C cultured with PD98059 using 607 western blot analysis. (E-H) Quantification of protein expression levels of Sema4C, 608 E-cadherin, p-ERK1/2, and total ERK (ERK1/2). (I) Intensity for p-ERK1/2 was 609 normalized to total ERK1/2 and represent as fold change over control group. ns, no 610 significant difference. 


\section{Figures}
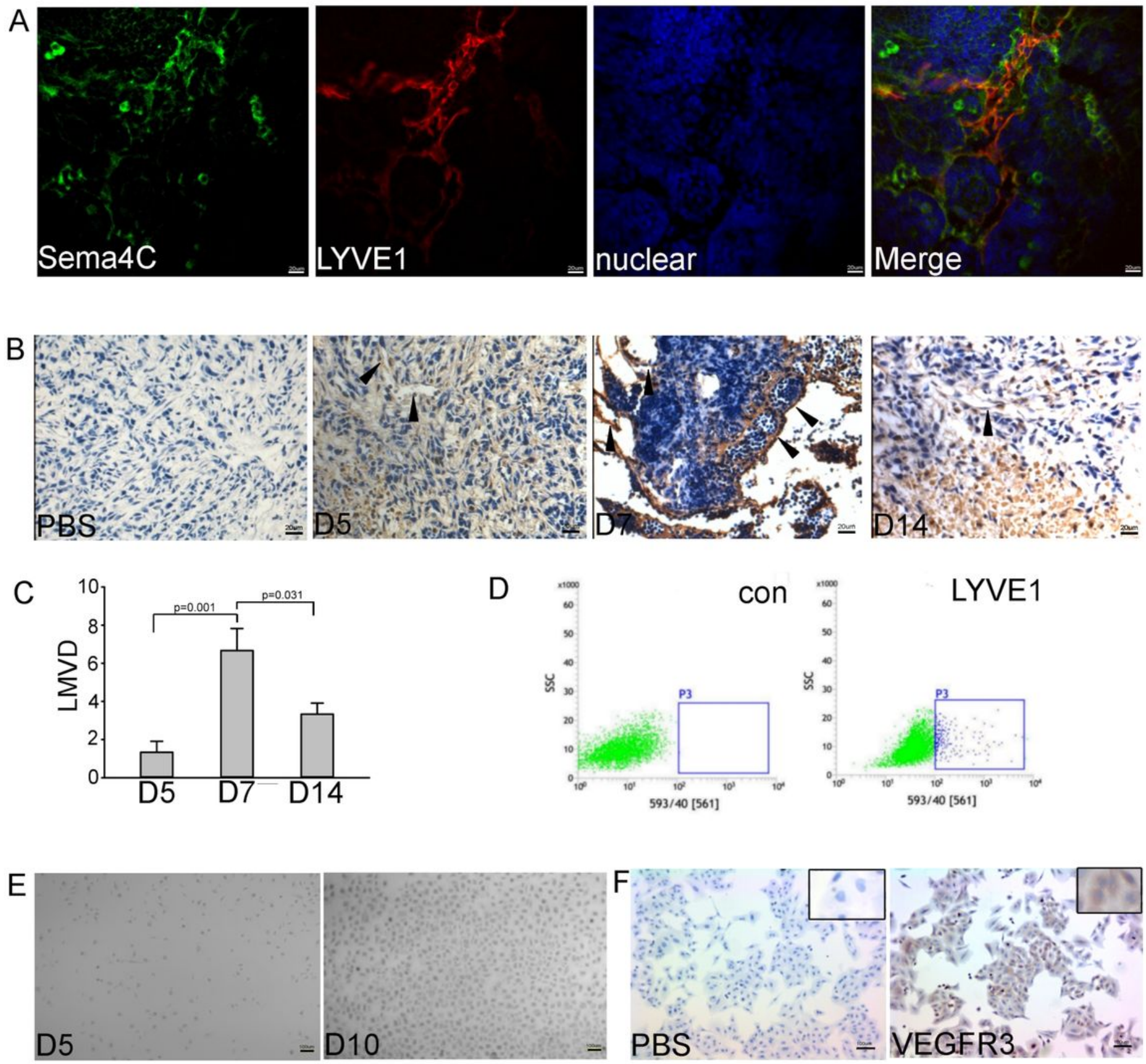

\section{Figure 1}

Tumor-associated lymphatic endothelial cells (TLECs)were obtained from a mouse xenograft tumor . (A) The location of Sema4C was detected in mouse tumor tissue. In lymphatic vessels, Sema4C(green) have co-location with LYVE1(Red), one of the most notable marker for lymphatic endothelial cells. Nuclei were shown by DAPI staining (blue). (B)The most suitable time (the 7th day after U14 cells injection) for separation of TLECs was determined by LYVE1 immunohistochemical analysis. (C) Quantification of the 
numbers of lymphatic vessels (LMVD) in 5th ,7th, 14th days were measured. (D)Tumor tissues were dissociated into a cell suspension and then stained for LYVE1-PE. LYVE1-positive cells were separated by flow cytometry. (E) TLECs were cultured in EBM-2 medium for 5 days and 10 days. (F) VEGFR3-positive TLECs were identified by immunohistochemistry.

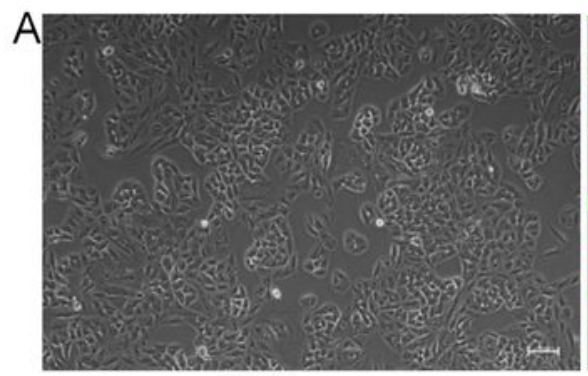

con

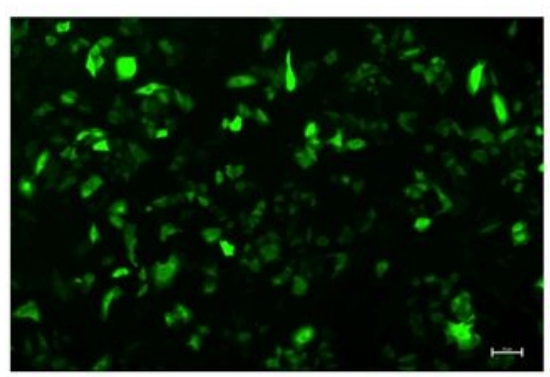

LV3-siRNA

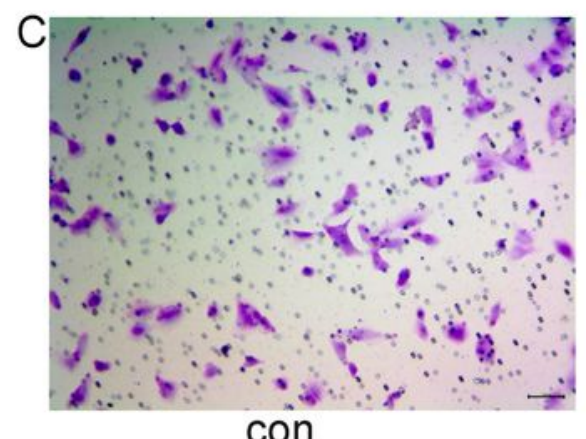
con

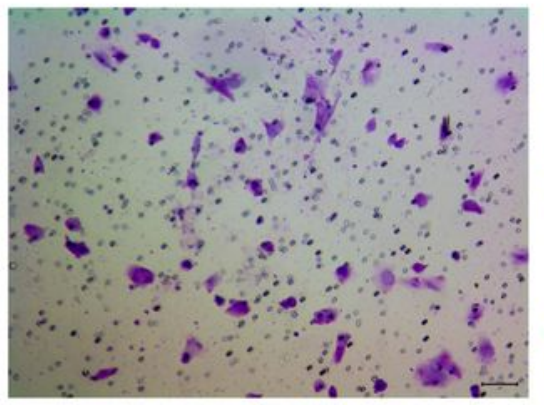

LV3-siRNA

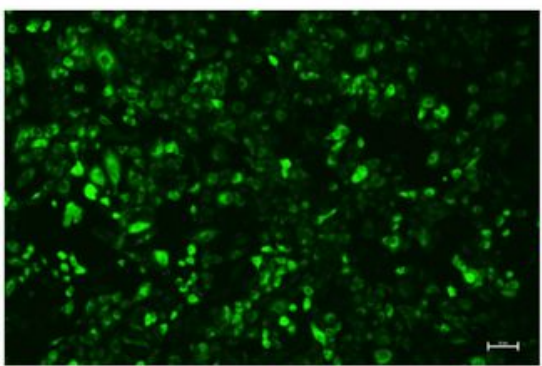

LV3NC

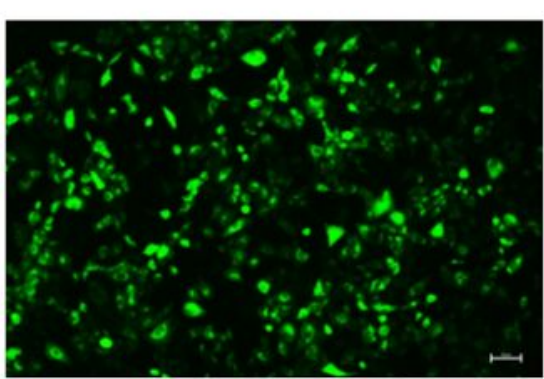

LV5-Sema4C

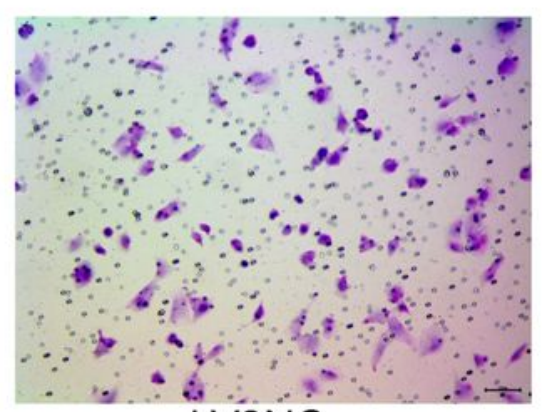

LV3NC

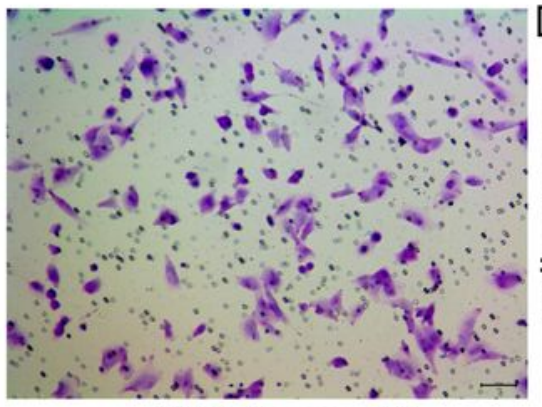

LV5-Sema4C

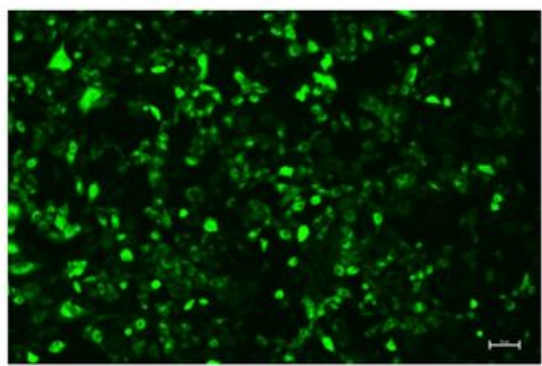

LV5NC
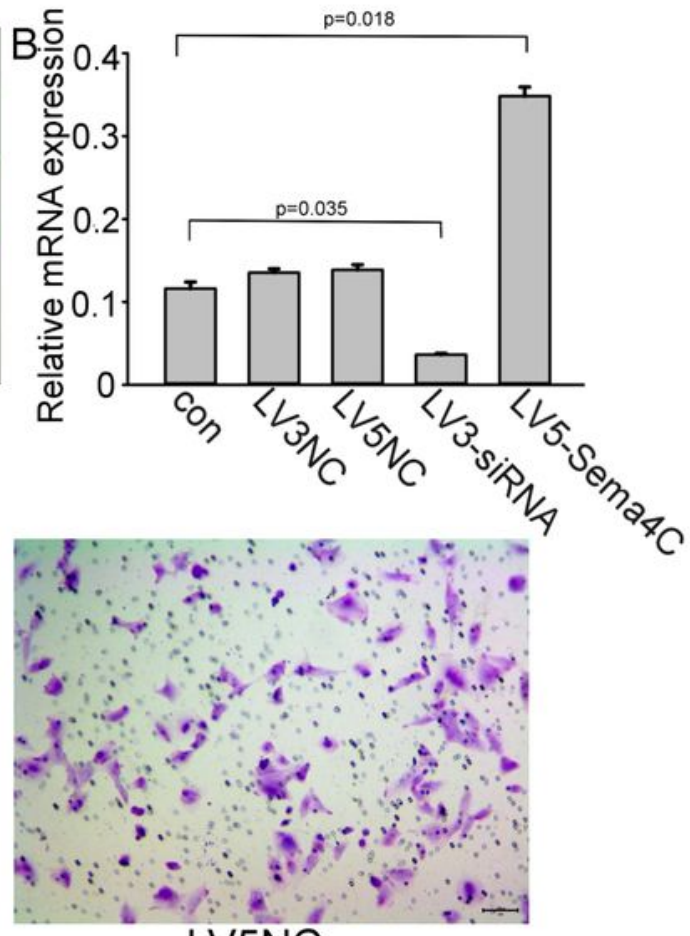

LV5NC

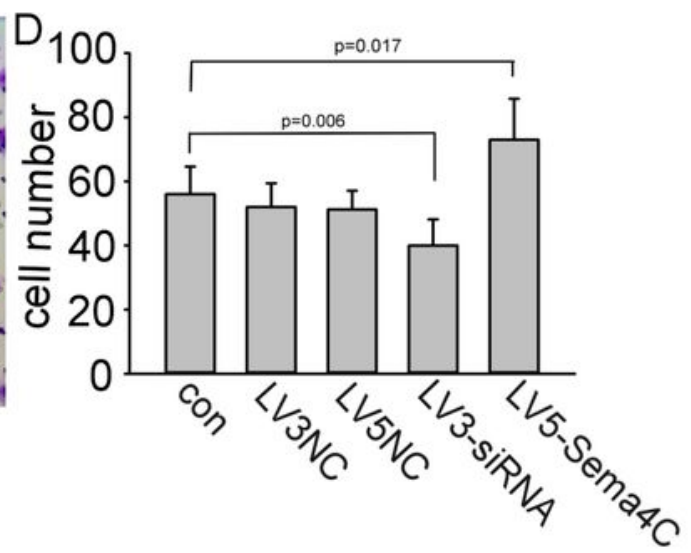

Figure 2 
Role of Sema4C expression on migration ability of TLECs. (A) TLECs treated with lentivirus medium only (con), lentivirus vector control for Sema4C siRNA (LV3NC), lentivirus vector control for full-length Sema4C (LV5NC), Sema4C siRNA (LV3-siRNA), and full-length Sema4C (LV5-Sema4C). (B) TLECs with LV3- siRNA or LV5-Sema4C were generated and confirmed by Sema4C mRNA expression as measured by real-time PCR. (C) The migration ability of cells (con, LV3NC,LV5NC,LV3-siRNA, and LV5-Sema4C) were assessed using a Transwell assay. (D) Quantification of the number of cells at the bottom of the Transwell chamber.
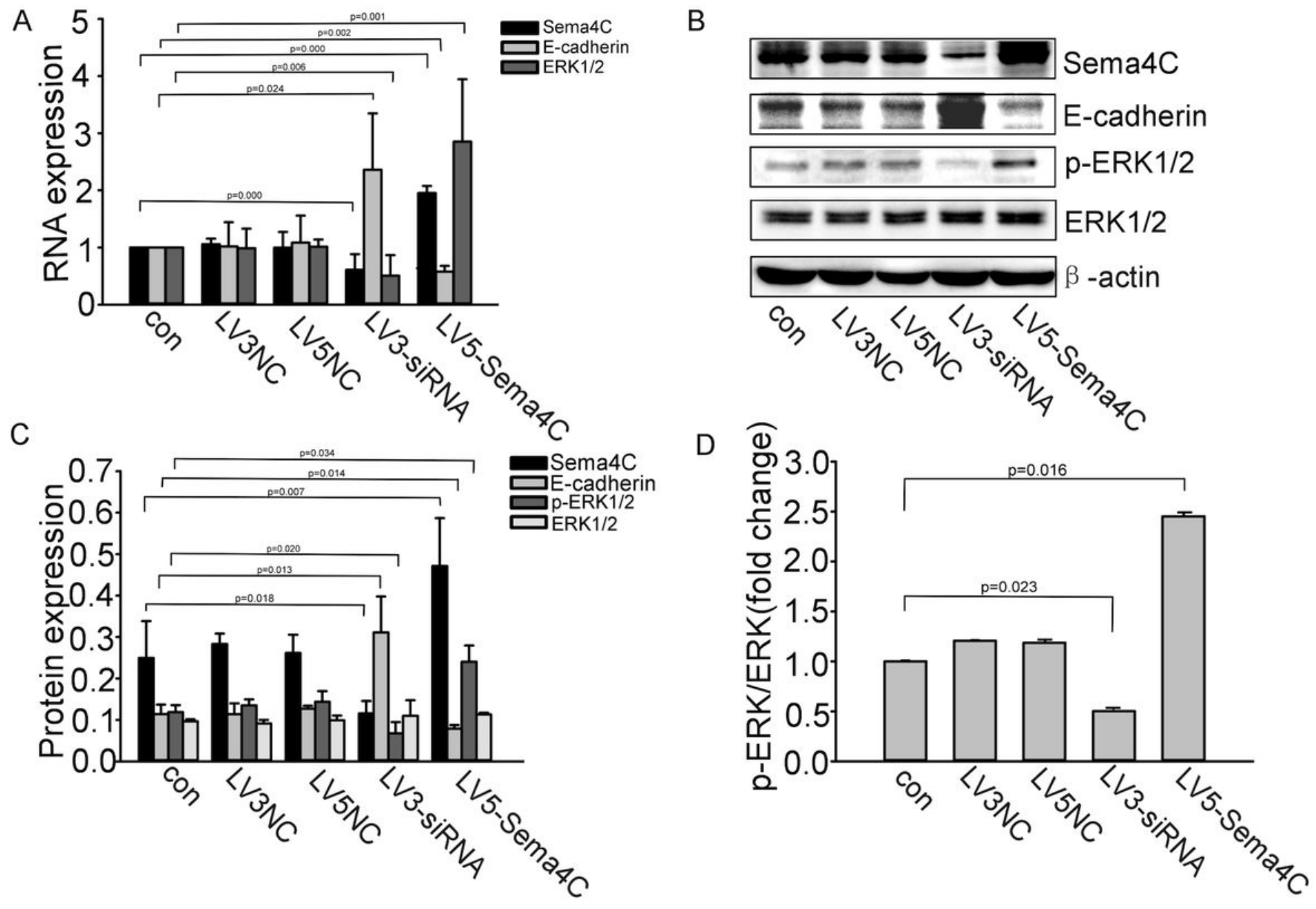

\section{Figure 3}

Role of Sema4C expression on EndMT of TLECs. (A) Assessment of mRNA expression levels of Sema4C, E-cadherin, and ERK1/2 using quantitative PCR. (B) Protein expression levels of Sema4C, E-cadherin, pERK1/2, and total ERK (ERK1/2) using western blot analysis. (C) Quantification of protein expression levels of Sema4C, E-cadherin, p-ERK1/2, and total ERK (ERK1/2) using western blot. (D) Intensity for pERK1/2 was normalized to total ERK1/2 and represent as fold change over control group. 


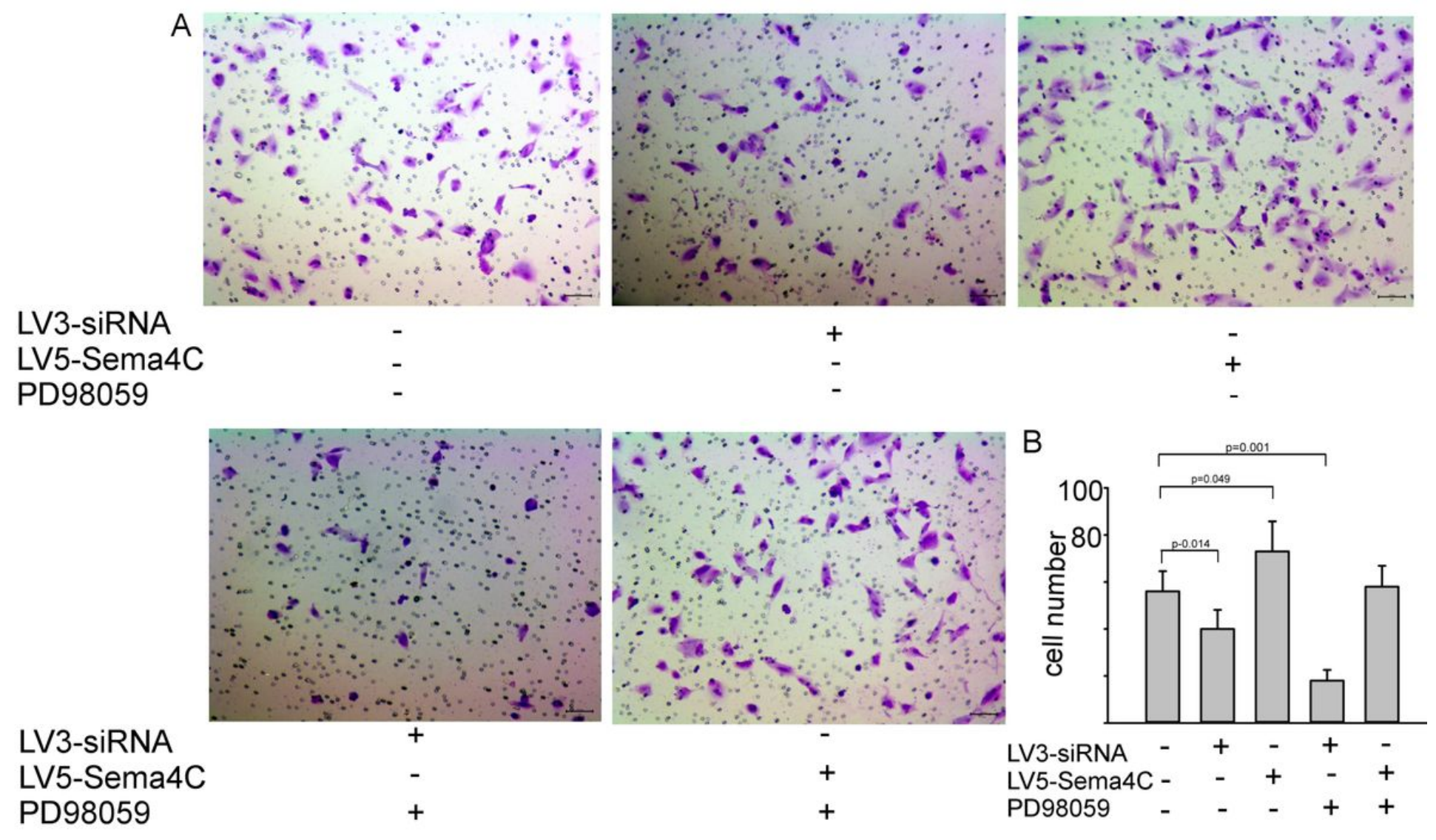

Figure 4

The ERK inhibitor can reverse migration ability of TLECs induced by Sema4C. (A) The migration ability in TLECs was assessed using only the media of lentivirus (con), Sema4C siRNA (LV3-siRNA), full-length Sema4C (LV5-Sema4C), LV3-siRNA cultured with PD98059, and LV5-Sema4C cultured with PD98059. (B) Quantification of the number of cells from the bottom of the Transwell inserts was measured. 

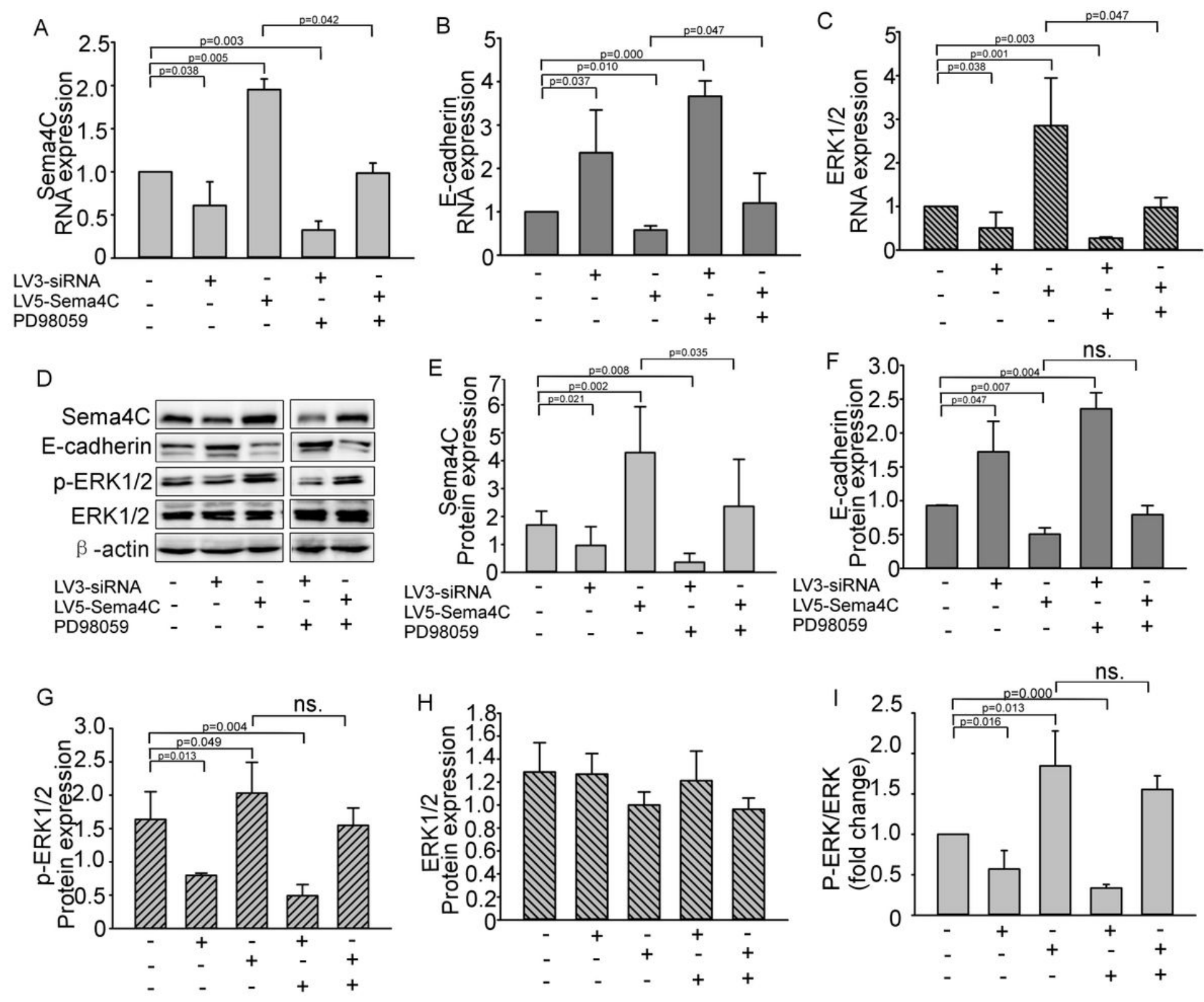

Figure 5

Sema4C stimulates EndMT of TLECs via the ERK pathway. (A-C) The RNA expression levels of Sema4C, E-cadherin, and total ERK (ERK1/2) in TLECs treated with only media of lentivirus (con), Sema4C siRNA (LV3-siRNA), full-length Sema4C (LV5-Sema4C), LV3-siRNA cultured with PD98059, and LV5-Sema4C cultured with PD98059. (D) The protein expression levels of Sema4C, E-cadherin, p-ERK1/2, and total ERK (ERK1/2) in TLECs treated with only media of lentivirus (con), Sema4C siRNA (LV3-siRNA), fulllength Sema4C (LV5-Sema4C), LV3- siRNA cultured with PD98059, and LV5-Sema4C cultured with PD98059 using western blot analysis. (E-H) Quantification of protein expression levels of Sema4C, Ecadherin, p-ERK1/2, and total ERK (ERK1/2). (I) Intensity for p-ERK1/2 was normalized to total ERK1/2 and represent as fold change over control group. ns, no significant difference. 